W. Kohnen

Nagoya Math. J.

Vol. 149 (1998), 83-92

\title{
FOURIER COEFFICIENTS AND HECKE EIGENVALUES
}

\author{
WINFRIED KOHNEN
}

\begin{abstract}
We will give certain asymptotic relations between $p$-eigenvalues and certain Fourier coefficients of Siegel cusp forms of genus $g$. In particular, it will turn out that potential strong bounds for the Fourier coefficients will imply potential strong bounds for the eigenvalues.
\end{abstract}

\section{$\S 1$. Introduction}

Let $F$ be a holomorphic non-zero cuspidal Hecke eigenform of integral weight $k$ on the Siegel modular group $\Gamma_{g}:=S p_{g}(\mathbf{Z})$ of genus $g$. We write $a(T)$ ( $T$ a positive definite half-integral matrix of size $g$ ) for the Fourier coefficients of $F$ and $\lambda_{p}$ ( $p$ a prime) for the eigenvalue of $F$ under the usual Hecke operator $T_{p}$.

For arbitrary $g \geq 2$ Fourier coefficients and eigenvalues are no longer proportional, and estimates for the first in general cannot be deduced from those for the latter. In the present paper we are interested in the converse question. In sect. 3 we shall prove that conjectural strong bounds for the $a(T)(\operatorname{det} T \rightarrow \infty)$ imply rather strong bounds for the $\lambda_{p}(p \rightarrow \infty)$. In particular, if $g=3$ a "generalized Ramanujan-Petersson conjecture" for the Fourier coefficients implies a "generalized Ramanujan-Petersson conjecture" for the eigenvalues (for a precise statement of these conjectures, cf. sect.2).

We therefore think that a closer study of the Fourier coefficients of Siegel cusp forms -so far somewhat neglected, as it seems- might be profitable.

Our result -in fact- is a refinement of an estimate for the difference $\lambda_{p} a(T)-a(p T)(T$ fixed $p \rightarrow \infty)$ given in [3,sect.1]. As in [3] the proof is rather ad hoc, however in contrast to [3] we need a much more detailled description of the action of $T_{p}$ on Fourier coefficients as given in [11].

Similar results as proved here can eventually be obtained also for the Hecke operators $T_{i, g-i}\left(p^{2}\right)(0 \leq i<g)$ which together with $T_{p}$ generate the

Received May 27, 1996. 
local Hecke algebra at $p$. One can also hope that they can be extended to Maass wave forms and -much more general- to cuspidal automorphic forms on arbitrary reductive linear algebraic groups.

\section{$\S 2$. Estimates for Fourier coefficients and eigenvalues}

Both for the Fourier coefficients and the eigenvalues of $F$ there are "generalized Ramanujan-Petersson conjectures" stating that

$$
a(T) \ll_{F, \epsilon}(\operatorname{det} T)^{\frac{k}{2}-\frac{g+1}{4}+\epsilon} \quad(\epsilon>0)
$$

and

$$
\lambda_{p} \ll_{g, \epsilon} p^{\frac{g k}{2}-\frac{g(g+1)}{4}+\epsilon} \quad(\epsilon>0),
$$

respectively. Conjecture (1) is due to Resnikoff-Saldaña [12] (the tribute to Ramanujan-Petersson here does not seem to be quite motivated) while (2) goes back to Satake [13], Kurokawa [9] and (in much more general context) to Langlands [10]. Actually (2) is an easy consequence of what is usually called the Ramanujan-Petersson conjecture for $F$, meaning that all the Satake $p$-parameters of $F$ are of absolute value 1 .

Using the analytic properties of the Rankin-Dirichlet series of $F$ and the well-known asymptotic bounds for class numbers due to Siegel and Kitaoka, one can show that (1) is best possible. One should expect that (2) is also best possible, but we do not know any proof of this.

Both conjectures in general are known to be wrong. If $g=2$, forms $F$ that are Saito-Kurokawa lifts give counterexamples both to (1) and (2), cf. $[1,4]$. Also if $8 \mid g$, there are certain theta series with spherical harmonics of weight $\frac{g}{2}+1$ whose Fourier coefficients do not satisfy (1), cf. [1]. Nevertheless, there is some hope that (1) and (2) generically should be true.

If $g=2$ and $k \geq 3$, recently Weissauer [15] proved the RamanujanPetersson conjecture in its stronger form for $F$ not a Saito-Kurokawa lift using the trace formula. For arbitrary $g \geq 2$ one knows according to FaltingsChai $[5$,chap.8,sect.5] that at least the product of the $p$-parameters of $F$ (suitably normalized) is of absolute value 1 .

Concerning the $\lambda_{p}$ 's, rather good estimates -however still far away from (2)- have been obtained for arbitrary $g \geq 2$ using either local representation theory [3] or methods from arithmetic algebraic geometry [7]. According to our information, the best bound so far available (under the assumption $k \geq g+1)$ is

$$
\lambda_{p} \ll_{g, \epsilon} p^{\frac{g k}{2}-\frac{g(g+2)}{8}}
$$


([7]; if $g$ is odd, a slightly better bound is proved).

Concerning estimates for the Fourier coefficients our knowledge at present is still extremely limited. After Hecke's bound

$$
a(T) \ll_{F}(\operatorname{det} T)^{\frac{k}{2}}
$$

the best result for arbitrary $g \geq 2$ (and $k>g+1$ ) at present seems to be

$$
a(T) \ll_{F, \epsilon}(\operatorname{det} T)^{\frac{k}{2}-\frac{1}{2 g}-\left(1-\frac{1}{g}\right) \beta_{g}+\epsilon} \quad(\epsilon>0)
$$

where

$$
\beta_{g}^{-1}:=4(g-1)+4\left[\frac{g-1}{2}\right]+\frac{2}{g+2}
$$

[2].

Also for $4 \mid g$ there are examples of forms $F$ of arbitrary large weights with

$$
a(T) \ll_{F, \epsilon}(\operatorname{det} T)^{\frac{k}{2}-\frac{1}{2}+\epsilon} \quad(\epsilon>0)
$$

$[8]$.

\section{$\S 3$. Statement of result}

In $[3$, sect.1] the estimate

$$
\lambda_{p} a(T)-a(p T) \ll_{F}(\operatorname{det} T)^{\frac{k}{2}} p^{\frac{g k}{2}-1}
$$

was shown. Let $\alpha \geq 0$ be fixed. Then as a consequence of (5) (choosing $T$ with $a(T) \neq 0$ ) one obtains

$$
\lambda_{p} \ll F p^{\frac{g k}{2}-\min \{1, g \alpha\}} \quad(p \rightarrow \infty)
$$

provided

$$
a(T) \ll_{F}(\operatorname{det} T)^{\frac{k}{2}-\alpha} \quad(\operatorname{det} T \rightarrow \infty) .
$$

The proof of (5) only uses the standard explicit description of the action of $T_{p}$ on Fourier coefficients together with the Hecke estimate (4) for the latter. Inspecting the proof and using the hypothesis (7) rather than (4), it is not difficult to improve somewhat upon the $p$-exponents in (5) and hence in (6). However, much better results can be obtained if one exploits a more detailled information of the action of $T_{p}$ as given in [11]. We shall prove 
ThEOREM. Let $\alpha \geq 0$ be fixed and suppose that the bound

$$
a(T) \ll_{F}(\operatorname{det} T)^{\frac{k}{2}-\alpha}
$$

holds for all $T$. Then one has

(9) $\quad \lambda_{p} a(T)-a(p T) \ll_{F}(\operatorname{det} T)^{\frac{k}{2}-\alpha} p^{\frac{g k}{2}-\kappa_{\alpha}} \quad(p>2, p \nmid \operatorname{det}(2 T))$

where

$$
\kappa_{\alpha}:= \begin{cases}g \alpha-(2 \alpha-2) & \left(0 \leq \alpha \leq \frac{3}{2}\right) \\ g \alpha-\left(\alpha-\frac{1}{2}\right)^{2} & \left(\alpha \geq \frac{3}{2}\right) .\end{cases}
$$

Observe that in the range $0 \leq \alpha \leq \frac{g+1}{4}$ the function $\alpha \mapsto \kappa_{\alpha}$ is positive and non-decreasing.

In the same way as before one deduces

COROLlary. Under the hypothesis (8) one has

$$
\lambda_{p} \ll_{F} p^{\frac{g k}{2}-\kappa_{\alpha}^{\prime}} \quad(p \rightarrow \infty)
$$

where

$$
\kappa_{\alpha}^{\prime}:= \begin{cases}g \alpha & (0 \leq \alpha \leq 1) \\ \kappa_{\alpha} & (\alpha \geq 1) .\end{cases}
$$

Note that if one should know (8) for some "large" $\alpha$ (i.e. $\alpha$ close to $\frac{g+1}{4}$ ), then by (10) one could considerably improve upon (3). It also follows from (10) that the bound (1) implies (2) if $g=3$.

In practice, however, as is clear from the discussion in sect. 2 the bounds for $\lambda_{p}$ so far truly provable using (10) are much weaker than those obtained previously by other tools.

\section{$\S 4$. Proof}

We use the notation

$$
A[B]=B^{\prime} A B
$$

for matrices $A$ and $B$ of appropriate sizes $\left(B^{\prime}=\right.$ transpose of $\left.B\right)$.

We abbreviate $\Gamma \cong \Gamma_{g}$. We let $J=\left(\begin{array}{cc}0 & E \\ -E & 0\end{array}\right)$ with 0 resp. $E$ the zero resp. unit matrix of size $g$. We denote by

$$
\mathcal{O}_{p}:=\left\{\gamma \in \mathbf{Z}^{(2 g, 2 g)} \mid J[\gamma]=p J\right\}
$$


the set of symplectic similitudes of scale $p$. For $\gamma=\left(\begin{array}{cc}A & B \\ C & D\end{array}\right) \in \mathcal{O}_{p}$ we put

$$
\left(\left.F\right|_{k} \gamma\right)(Z):=(\operatorname{det} \gamma)^{\frac{k}{2}} \operatorname{det}(C Z+D)^{-k} F\left((A Z+B)(C Z+D)^{-1}\right)
$$

( $Z$ in the Siegel upper half-space of genus $g$ ).

Then by definition

$$
T_{p} F=\left.p^{\frac{g k}{2}-\frac{g(g+1)}{2}} \sum_{\gamma \in \Gamma \backslash \mathcal{O}_{p}} F\right|_{k} \gamma
$$

As is well-known, a set of representatives for $\Gamma \backslash \mathcal{O}_{p}$ can be chosen in upper triangular form $\left(\begin{array}{cc}A & B \\ 0 & D\end{array}\right)$. More precisely, when $D$ runs over a fixed set of left non-associated (w.r.t. $G L_{g}(\mathbf{Z})$ ) right-divisors of $p E$ and one sets $A=p D^{\prime-1}$, and moreover $B$ runs over a set of representatives of matrices in $\mathbf{Z}^{(g, g)}$ satisfying $B^{\prime} D=D^{\prime} B$ modulo the equivalence relation $B_{1} \sim B_{2}$ if $B_{1}-B_{2}=S D$ with $S \in \mathbf{Z}^{(g, g)}, S^{\prime}=S$, then $\left(\begin{array}{cc}A & B \\ 0 & D\end{array}\right)$ runs over a set of representatives for $\Gamma \backslash \mathcal{O}_{p}[6$, chap.IV, sects.2,3]. Therefore

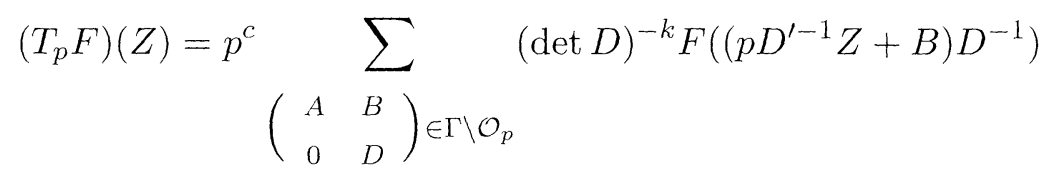

where

$$
c:=g k-\frac{g(g+1)}{2} .
$$

Inserting the Fourier expansion

$$
F(Z)=\sum_{T>0} a(T) e^{2 \pi i \operatorname{tr}(T Z)}
$$

we obtain

$$
=p^{c} \underset{T>0,\left(\begin{array}{cc}
A & B \\
0 & D
\end{array}\right) \in \Gamma \backslash \mathcal{O}_{p}}{\left(T_{p} F\right)(Z)} a(T)(\operatorname{det} D)^{-k} e^{2 \pi i \operatorname{tr}\left(T B D^{-1}\right)} e^{2 \pi i \operatorname{tr}\left(p T\left[D^{\prime-1}\right] Z\right)}
$$


We now follow [11] (one could also consult [6, chap.IV, sect.3]). According to $[11$, Satz 6$]$ we can choose

$$
\left(\begin{array}{cc}
A & B \\
0 & D
\end{array}\right)=\left(\begin{array}{cc}
p \Delta^{-1} & G \\
0 & \Delta
\end{array}\right)\left(\begin{array}{cc}
U^{-1} & 0 \\
0 & U^{\prime}
\end{array}\right)
$$

with $\Delta, U$ and $G$ as follows:

- $\Delta=\operatorname{diag}\left[d_{1} d_{2} \ldots d_{g}\right]$ is an integral diagonal matrix with $d_{1}\left|d_{2}\right| \cdots\left|d_{g}\right| p$, $d_{\mu}>0(\forall \mu)$;

- for $\Delta$ fixed, $U$ runs over a set of representatives for $G L_{g}(\mathbf{Z}) / G L_{g, \Delta}(\mathbf{Z})$ where $G L_{g, \Delta}(\mathbf{Z}):=G L_{g}(\mathbf{Z}) \cap \Delta G L_{g}(\mathbf{Z}) \Delta^{-1}$;

- $G=\left(g_{\mu \nu}\right)$ runs over all matrices in $\mathbf{Z}^{(g, g)}$ such that $g_{\mu \nu}=\frac{d_{\nu}}{d_{\mu}} g_{\nu \mu}(\mu<$ $\nu)$ and $0 \leq g_{\mu \nu}<d_{\nu}(\mu \geq \nu)$.

Inserting into (12) one picks up the exponential sum

$$
\begin{aligned}
& \sum_{G} e^{2 \pi i \operatorname{tr}\left(T G \Delta^{-1}\right)} \\
& \quad= \begin{cases}\prod_{\nu=1}^{g} d_{\nu}^{g+1-\nu} & \text { if } d_{\mu}\left|t_{\mu \mu} \forall \mu, d_{\nu}\right| 2 t_{\mu \nu} \forall \mu, \nu \text { with } \mu>\nu \\
0 & \text { otherwise }\end{cases}
\end{aligned}
$$

$\left(T=\left(t_{\mu \nu}\right)\right)$. If $b(T)$ denotes the $T$-th Fourier coefficient of $T_{p} F$, one obtains from this easily

$$
b(T)=p^{c} \sum_{\Delta, U}\left(\prod_{\nu=1}^{g} d_{\nu}^{g+1-\nu-k}\right) a\left(\frac{1}{p} T[U \Delta]\right)
$$

with $\Delta$ as before and $U$ for fixed $\Delta$ running over elements in $G L_{g}(\mathbf{Z}) / G L_{g, \Delta}(\mathbf{Z})$ such that

$$
\frac{1}{p} T[U \Delta]=\left(\begin{array}{ccccc}
d_{1} s_{11} & d_{1} s_{12} & d_{1} s_{13} & \ldots & d_{1} s_{1 g} \\
d_{1} s_{21} & d_{2} s_{22} & d_{2} s_{23} & \ldots & d_{2} s_{2 g} \\
\vdots & \vdots & \vdots & \ddots & \vdots \\
d_{1} s_{g 1} & d_{2} s_{g 2} & d_{3} s_{g 3} & \ldots & d_{g} s_{g g}
\end{array}\right)
$$

where $S=\left(s_{\mu \nu}\right) \in \mathbf{Z}^{(g, g}$ is half-integral (and symmetric). 
So far the above computations would have been valid with $p$ replaced by an arbitrary positive integer. We shall now use that $p$ is a prime. In this case we have

$$
d_{1}=\ldots=d_{r}=1, d_{r+1}=\ldots=d_{g}=p
$$

for some $r \in\{0,1, \ldots, g\}$. If $r=0$ or $r=g$ one has $\Delta=\operatorname{diag}[p, \ldots, p]$ or $\Delta=E$, respectively, and $G L_{g, \Delta}(\mathbf{Z})=G L_{g}(\mathbf{Z}), U=E$. If $r \in\{1, \ldots, g-1\}$ then according to the arguments given in [11, pp.103-105] one can choose as a set of representatives for $G L_{g}(\mathbf{Z}) / G L_{g, \Delta}(\mathbf{Z})$ the matrices $(Q R)$ where $Q$ runs over a set of representatives for matrices in $(\mathbf{Z} / p \mathbf{Z})^{(g, r)}$ having maximal rank $\bmod p$, modulo right multiplication by $G L_{r}(\mathbf{Z} / p \mathbf{Z})$, and each such $Q$ is completed in exactly one way to an element $(Q R)$ in $G L_{g}(\mathbf{Z})$. The condition (14) then is equivalent to $T[Q] \equiv 0(\bmod p)$.

From the condition (14) we see, in particular, that the value

$$
\operatorname{det}\left(2 \cdot \frac{1}{p} T[U \Delta]\right)=p^{g-2 r} \operatorname{det}(2 T)
$$

is integral, hence since by assumption $p \nmid \operatorname{det}(2 T)$, we conclude that only matrices $\Delta_{r}=\operatorname{diag}\left[d_{1} \ldots d_{r} d_{r+1} \ldots d_{g}\right]$ with $r \in\left\{0,1, \ldots,\left[\frac{g}{2}\right]\right\}$ can contribute to the sum on the right of (13). The term with $r=0$ gives the contribution

$$
\left(\prod_{\nu=1}^{g} p^{g+1-\nu-k}\right) a(p T)
$$

hence its contribution to $b(T)$ is $a(p T)$.

Since $T_{p} F=\lambda_{p} F$ we therefore obtain

$$
\begin{aligned}
\lambda_{p} a(T)-a(p T) & =p^{c} \sum_{r=1}^{\left[\frac{g}{2}\right]} \sum_{U}\left(\prod_{\nu=r+1}^{g} p^{g+1-\nu-k}\right) a\left(\frac{1}{p} T\left[U \Delta_{r}\right]\right) \\
& \ll F p^{c} \sum_{r=1}^{\left[\frac{g}{2}\right]} \sum_{U}\left(\prod_{\nu=r+1}^{g} p^{g+1-\nu-k}\right)\left(\operatorname{det}\left(\frac{1}{p} T\left[U \Delta_{r}\right]\right)\right)^{\frac{k}{2}-\alpha}
\end{aligned}
$$

where in the last line we have used (8).

Since

$$
\sum_{\nu=r+1}^{g}(g+1-\nu-k)=\frac{g(g+1)}{2}-g k-r(g+1-k)+\frac{r(r+1)}{2}
$$


and

$$
\operatorname{det}\left(\frac{1}{p} T\left[U \Delta_{r}\right]\right)=p^{g-2 r} \operatorname{det} T
$$

it follows (observing (11)) that

$$
\lambda_{p} a(T)-a(p T) \ll_{F}(\operatorname{det} T)^{\frac{k}{2}-\alpha} p^{g\left(\frac{k}{2}-\alpha\right)} \sum_{r=1}^{\left[\frac{g}{2}\right]} p^{r\left(2 \alpha-g+\frac{r-1}{2}\right)} N_{r, T}
$$

where

$$
N_{r, T}:=\#\left\{Q \in \mathbf{F}_{p}^{(g, r)} \mid \operatorname{rk} Q=r, T[Q]=0\right\} / G L_{r}\left(\mathbf{F}_{p}\right)
$$

(here we have identified $T \in \frac{1}{2} \mathbf{Z}^{(g, g)}$ with its image in $\mathbf{F}_{p}^{(g, g)}$ ).

Lemma. Let $r \in\left\{1, \ldots,\left[\frac{g}{2}\right]\right\}$. Then $N_{r, T} \ll g p^{r(g-r)-\frac{r(r+1)}{2}}$.

Proof. Since $p \neq 2$, we may assume that $T$ is diagonal. Note that $T$ is regular by hypothesis.

Any $Q \in \mathbf{F}_{p}^{(g, r)}$ with $\operatorname{rk} Q=r$ is equivalent modulo right-multiplication by elements in $G L_{r}\left(\mathbf{F}_{p}\right)$ and simultaneous left-mutiplication by permutation matrices in $G L_{g}\left(\mathbf{F}_{p}\right)$ to a matrix of the form $\left(\begin{array}{c}E_{r} \\ X\end{array}\right)$ with $X \in \mathbf{F}_{p}^{(g-r, r)}$. Let $P \in G L_{g}\left(\mathbf{F}_{p}\right)$ be a permutation matrix. If $T[P]=\tilde{T}=\left(\begin{array}{cc}\tilde{T}_{1} & 0 \\ 0 & \tilde{T}_{2}\end{array}\right)\left(\tilde{T}_{1} \in\right.$ $\left.\mathbf{F}_{p}^{(r, r)}, \tilde{T}_{2} \in \mathbf{F}_{p}^{(g-r, g-r)}\right)$, then $\tilde{T}\left[\left(\begin{array}{c}E_{r} \\ X\end{array}\right)\right]=0$ means that $\tilde{T}_{2}[X]=-\tilde{T}_{1}$.

Therefore it is sufficient to prove that for $T_{1} \in G L_{r}\left(\mathbf{F}_{p}\right), T_{2} \in G L_{g-r}\left(\mathbf{F}_{p}\right)$ one has

$$
N_{r, T_{1}, T_{2}}:=\#\left\{X \in \mathbf{F}_{p}^{(g-r, r)} \mid T_{2}[X]=-T_{1}\right\} \ll_{g} p^{r(g-r)-\frac{r(r+1}{2}} .
$$

According to [14, Hilfssatz 12], under the hypothesis $T_{1}, T_{2}$ invertible and $p \neq 2$ one has

$$
= \begin{cases}\left(1-\delta_{1} p^{-\frac{g-r}{2}}\right)\left(1+\delta_{2} p^{r-\frac{g}{2}}\right) \prod_{j=1}^{\frac{r}{2}-1}\left(1-p^{2 j-(g-r)}\right) & \text { if } g \text { even, } r \text { even } \\ \left(1-\delta_{1} p^{-\frac{g-r}{2}}\right) \prod_{j=1}^{\frac{r-1}{2}}\left(1-p^{2 j-(g-r)}\right) & \text { if } g \text { odd, } r \text { odd } \\ \left(1+\delta_{2} p^{r-\frac{g}{2}}\right) \prod_{j=1}^{\frac{r-1}{2}}\left(1-p^{2 j-(g-r)-1}\right) & \text { if } g \text { even, } r \text { odd } \\ \prod_{j=1}^{\frac{r}{2}}\left(1-p^{2 j-(g-r)-1)}\right) & \text { if } g \text { odd, } r \text { even }\end{cases}
$$


where

$$
\begin{aligned}
& \delta_{1}:=\left(\frac{(-1)^{\frac{g-r}{2}} \operatorname{det} T_{2}}{p}\right) \quad(g-r \text { even }), \\
& \delta_{2}:=\left(\frac{(-1)^{\frac{g}{2}-r} \operatorname{det} T_{1} \operatorname{det} T_{2}}{p}\right) \quad(g \text { even }) .
\end{aligned}
$$

Since $r \leq \frac{g}{2}$, each single factor on the right of $(15)$ is $\leq 2$ or $\leq 1$, respectively. This proves the Lemma. From the Lemma we now obtain

$$
\lambda_{p} a(T)-a(p T) \ll_{F}(\operatorname{det} T)^{\frac{k}{2}-\alpha} p^{g\left(\frac{k}{2}-\alpha\right)} \sum_{r=1}^{\left[\frac{g}{2}\right]} p^{r(2 \alpha-1-r)} .
$$

If $0 \leq \alpha \leq 1$, then all terms in the sum on the right of (16) are $\leq 1$, and the largest term is $p^{2 \alpha-2}$.

For $\alpha \geq 1$ we write

$$
\sum_{r=1}^{\left[\frac{g}{2}\right]} p^{r(2 \alpha-1-r)}=\sum_{1 \leq r<2 \alpha-1} p^{r(2 \alpha-1-r)}+\mathrm{O}_{g}(1),
$$

and the right-hand side is

$$
\ll_{g} \begin{cases}p^{2 \alpha-2} & \text { if } \alpha \leq \frac{3}{2} \\ p^{\left(\alpha-\frac{1}{2}\right)^{2}} & \text { if } \alpha \geq \frac{3}{2}\end{cases}
$$

by some elementary considerations. This proves the theorem.

\section{REFERENCES}

[1] Böcherer, S. and Raghavan, S., On Fourier coefficients of Siegel modular forms, J. Reine Angew. Math., 384 (1988), 80-101.

[2] Böcherer, S. and Kohnen, W., Estimates for Fourier coefficients of Siegel cusp forms, Math. Ann., 297 (1993), 499-517.

[3] Duke, W., Howe, R. and Li, J.-S., Estimating Hecke eigenvalues of Siegel modular forms, Duke Math. J., 67, no.1 (1992), 219-240.

[4] Eichler, M. and Zagier, D., The theory of Jacobi forms, Progr. Math., 55 (1985). 
[5] Faltings, G. and Chai, Ch.-L., Degeneration of abelian varieties, Ergebnisse d. Math., 22, Berlin Heidelberg New York: Springer 1990.

[6] Freitag, E., Siegelsche Modulformen, Berlin Heidelberg New York: Springer 1983.

[7] Hatada, K., Estimates for eigenvalues of Hecke operators on Siegel cusp forms, J. Reine Angew. Math., 480 (1996), 105-123.

[8] Kohnen, W., On Siegel modular forms, Compos. Math, 103 (1996), 219-226.

[9] Kurokawa, N., Examples of eigenvalues of Hecke operators on Siegel cusp forms of degree two, Invent. Math., 49 (1978), 149-165.

[10] Langlands, R.P., Problems in the theory of automorphic forms, Lect. Not. in Modern Analysis and Appl. III, pp. 18-61.

[11] Maass, H., Die Primzahlen in der Theorie der Siegelschen Modulformen, Math. Ann., 124 (1951), 87-122.

[12] Resnikoff, H.L. and Saldaña, R.L., Some properties of Fourier coefficients of Eisenstein series of degree two, J. Reine Angew. Math., 265 (1974), 90-109.

[13] Satake, I., Spherical functions and Ramanujan conjecture, Algebraic groups and discontinuous subgroups (Borel, A. and Mostow, G.P., eds.), pp. 258-264.

[14] Siegel, C.L., Über die analytische Theorie der quadratischen Formen, Collected Works I (Chandrasekharan, K. and Maass, H., eds.), Springer, Berlin Heidelberg New York (1966), pp. 326-405.

[15] Weissauer, R., The Ramanujan conjecture for genus two Siegel modular forms (an application of the trace formula), Preprint 1993.

\author{
Mathematisches Institut \\ Universität Heidelberg \\ Im Neuenheimer Feld 288 \\ 69120 Heidelberg \\ Germany \\ winfried@mathi.uni-heidelberg.DE
}

